\title{
CREATIVITY AND TECHNOLOGY IN MATHEMATICS: FROM STORY Telling TO AlgORITHMic WiTh OP'ART
}

\author{
Christian Mercat, Pedro Lealdino Filho, Mohamed El-Demerdash
}

\begin{abstract}
This article describes some of the results of the European project mcSquared (http://mc2-project.eu/) regarding the use of Op'Art and optical illusion pieces as a tool to foster modeling and creative mathematical thinking in students. We present briefly the c-book technology and some results we got experimenting it. The Op'Art movement, with artists such as Victor Vasarely, Julio Le Parc or Bridget Riley, uses algorithms to create geometric patterns. It is a goldmine for mathematical thinking, from early ages, with the use of narratives to describe the paintings and produce new ones in a controlled way, up to higher education with generative art produced by the implementation of algorithm. The outcome is that technology, far from hindering the creative process actually can be used to help putting it on firm grounds, narrowing the diverging phase into a converging one by giving means to objectify the description and creation of the artwork itself and making explicit the choices made, allowing for the creative exploration of new original ways: "what if...". This objectivation is done differently by different audiences but comprises the same phases of identifying the relevant variables (disks, squares, colors, position), describing their relationships (the color of the disks depends on their position in such a way) and exploring the space of configurations the modeling gives rise to.
\end{abstract}

Keywords: Mathematics Education, Creativity, Creative Mathematical Thinking, Digital Resources, Technology Enhanced Learning, Op Art

\section{Introduction}

If mathematics is the queen of science, geometry is the queen of mathematics. Unfortunately, this paramount subject has not been well treated in recent curricula reforms in Europe. However, the appearance of algorithmic and programming therein allow for practical rather than theoretical geometry. A great divide between geometry approaches in primary and secondary schools is the change from a perceptive to an axiomatic geometry, from touched and seen objects to relations between concepts. In primary school mathematics, using the ruler is enough to prove that three points are aligned. This article describes an attempt at finding a pathway to ease this process through narration, from early personal and subjective storytelling to programming, and relates how Art and Technology come as perfect tools for this objective. Understanding the need for axiomatization can be fostered by description of figures with the explicit goal that someone else can, out of this description, reproduce a similar one. Optical Illusions are very interesting in that respect because what is seen is not exactly what is actually drawn. For example lines are parallel while they don't look so in Figure. 1. As a construction protocol, it makes explicit the difference between a figure and a drawing (Parzysz, Duval, Laborde-Capponi), paving the way for an axiomatic approach to geometry. In this article we focus our attention to simple series and sequences, not individual elaborate drawings: the complexity does not rely on many degrees of freedom but on an algorithm. Therefore the complexity can be reduced, the underlying scheme can be described, the figure wants to tell you a story, something begs to be understood, and the user knows when he has understood it. 


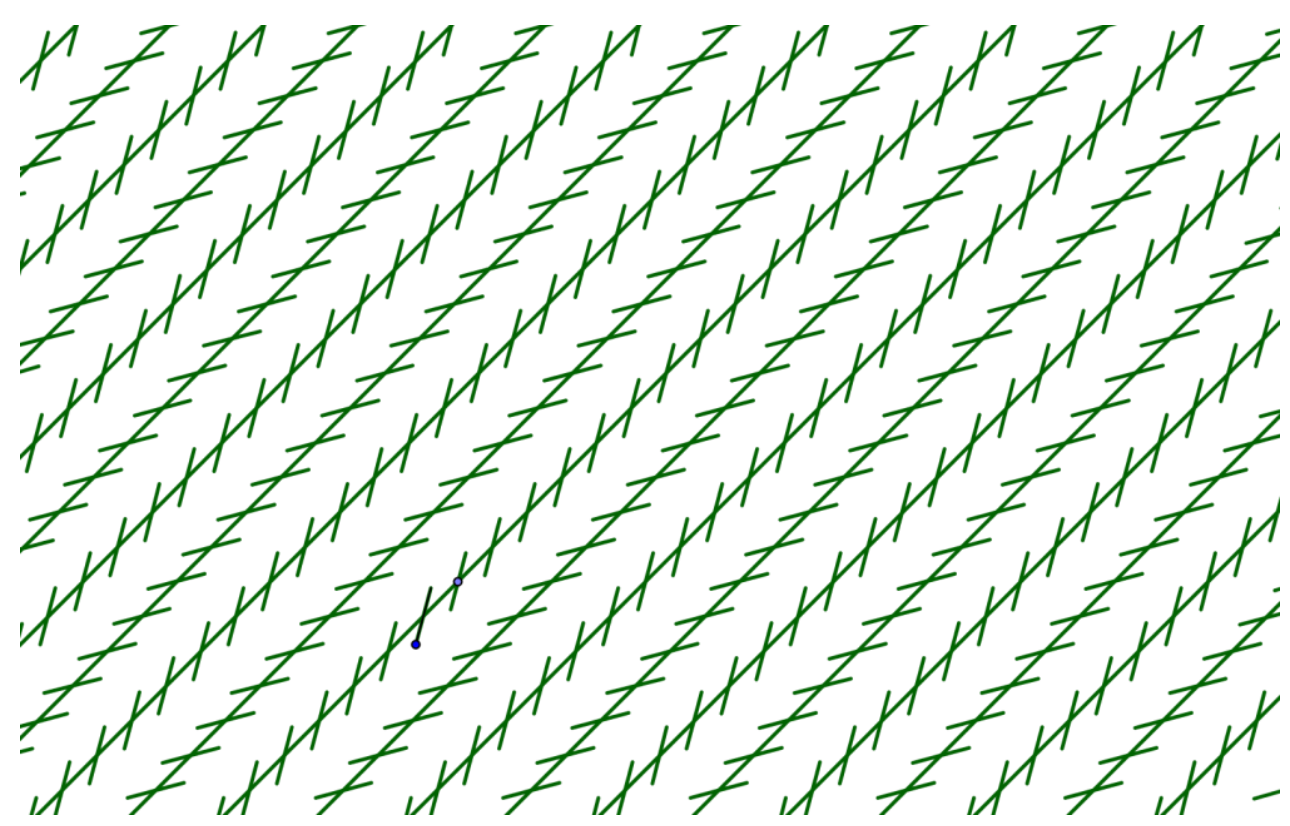

Figure 1. The Railway Illusion. Notice the two dots allowing for exploration: Unlike a static reproduction, the distance between segments, their length and orientation can be modified and the configuration space explored.

Deconstruction of complex figures has been used to study many aspects of cognition such as perception and memory (Rey, 1941, Osterrieth 1944), stereotype threat between girls and boys (Huguet-Régner, 2007) visualization and perception (Duval, 2005), geometric paradigms (HoudementKuzniak, 2006) or functions of drawings (Chaachoua, 1997). The use of interactive geometry as a mean to explore complex configuration spaces and as a lever towards abstraction has been pointed out many times (Laborde-Sträÿer, 2010), but linking it to narratives or language issues (Bulf-MathéMithalal, 2015) and even more interactive storytelling is a rather recent phenomenon: The M C Squared project (see acknowledgments) created the C-book technology, an infrastructure allowing for the blending of hyper-text, images and widgets of diverse origins into a single interactive narrative, an electronic book aimed at promoting creative mathematical thinking in its users. The description of the technological infrastructure of this project is not the focus of this article, suffice to say that students can create and share pictures, programs and interactive geometry figures of different origins, and discuss about them on the same page, accumulate these pages in an electronic book. Many different cbook units (about 70) have been produced in this project and this article reports on the usage of the cbook Art and Modeling created by the French Community of Interest. This c-book has been used in different contexts, and we report here on 2 pre-service teacher training sessions (45 students) and a workshop in the international congress of IREM network, Strasbourg, July 2016 (30 teachers, teachertrainers and researchers). The data collected and discussed here are their electronic productions only but the exchanges and interplays were not recorded, our interpretation is nevertheless discussed here.

\section{The Op'Art Movement}

In 1969 in Chicago, Arman, Armajani, Le Witt, Morris, Serra and other artists participated in an Art by Telephone exhibition: artwork was actually performed by someone (or a computer) following instructions dictated over a phone. The Op'Art movement is along the same lines. Unlike figurative art, it is rather minimalist in its approach: most productions can be described explicitly by a few sentences and yet the result is very compelling. It often uses anomalous motion optical illusions to create static but virtually vibrant compositions. Its main artists are Victor Vasarely, the G.R.A.V. artists, Julio Le Parc, Garcia Rossi, François Morellet, Francisco Sobrino, Stein, Jean-Pierre Vasarely (aka Yvaral), and Bridget Riley. Denise René gallery hosted and promoted these painters in Paris. The c-book uses as well the work of Prof. Akiyoshi Kitaoka (Kitaoka 2014). Contemporary trends such as generative or algorithmic art movements can be linked to the Op'Art movement. 


\section{From Subjective Storytelling to Algorithm}

In the c-book, users are presented with variations inspired by the work of Op'Art artists and optical illusions. In face to face experiments, both color printed handouts and interactive numeric versions are used. The tasks of describing the chosen piece are scaffolded by having to describe it in several steps, in writing, for oneself, to someone else, on the phone so that the person can reproduce it, and in actual roles playing circumstances, in several rows as an emitter or a receiver, orally or through the epsilonchat facility. In a constructionist approach, half-baked worlds with color pickers (see Figure. 2), unfinished or unrelated bits, are available for scaffolding the final implementation.

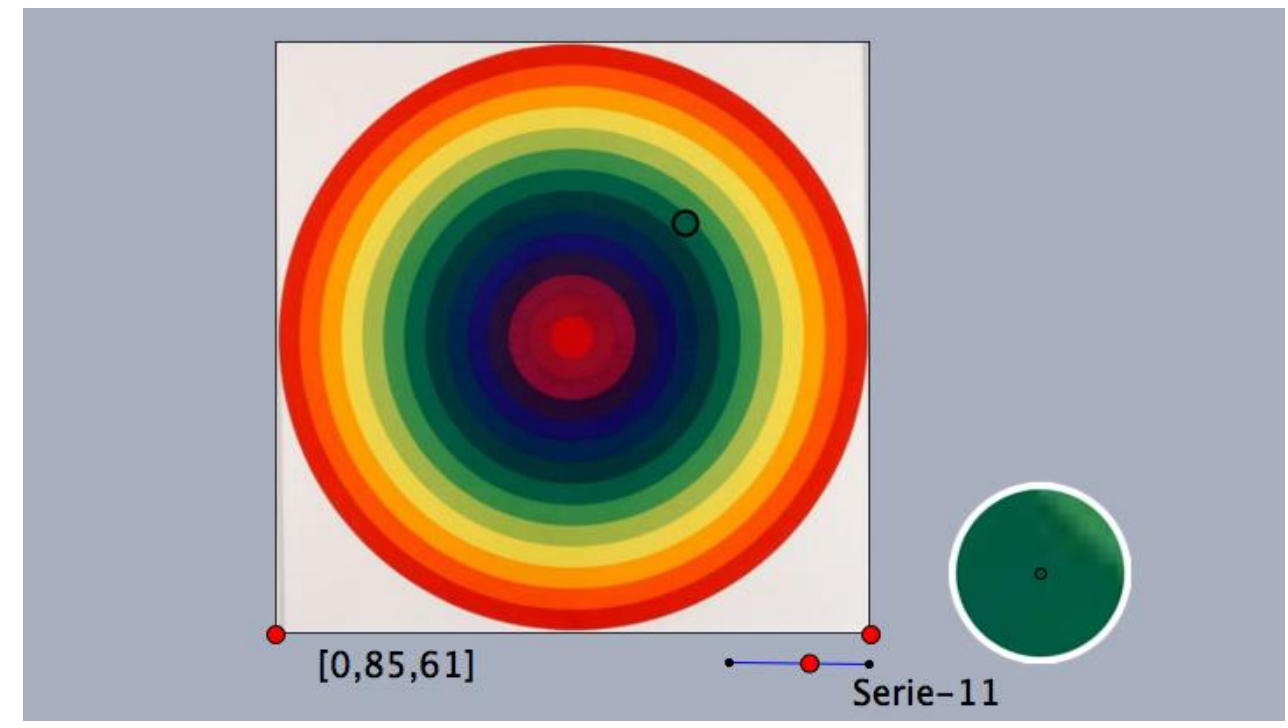

Figure 2. Color-picking dark green $(R=0, G=85, B=61)$ in Julio Le Parc Composition using Cinderella (DGS).

Reproducing a figure is a creative process. It requires modeling its construction, identifying its main components and the way they are related to one another. Moreover, once a specific creation has been understood, the choices made for that particular instantiation are explicit and lead to the conceptualization of the configuration space of further creations. Observing the production of artists such as Julio Le Parc over the years is really interesting in that respect: sticking to one particular color progression, he spatialized it in many different ways, slowly evolving, as uniformly changing concentric rings, to changing in pairs, in spirals, in disks and squares... Creativity is sometimes about playing with the limits, acknowledging them and going beyond in a controlled way. This activity is allowing the users to see in mathematics a powerful tool to express and control their exploration.

Some aspects of Creative Mathematical Thinking can be modeled by the Creativity Diamond (Figure. 3), born from networking strategies (Prediger et Al., 2008) combining and integrating several theories on creativity and mathematical thinking. This conceptual framework describes the creative process when someone attempts to solve mathematical problems. As seen before on the example of Julio le Parc, creating a figure is a creative process and the figure itself is a creative product.

Guilford emphasized the distinction between convergent and divergent thinking. He introduced the model of "Divergent Thinking" as the main ingredient of creativity. Drawing on his model (Guilford, 1950), the generation of new ideas shows the abilities of fluency, flexibility, originality and elaboration in mathematics that are defined as follows (El-Demerdash et al., 2016):

- Fluency means the individual's ability to pose or come up with many mathematical ideas or configurations related to a mathematical problem or situation in a short time.

- Flexibility refers to the individual's ability to vary the approach or suggest a variety of different methods toward a mathematical problem or situation.

- Originality means the individual's ability to try novel or unique approaches toward a mathematical problem or situation. 
- Elaboration is the individual's ability to redefine a single mathematical problem or situation to create others, going deeper, giving elaborated solutions, by changing one or more aspects by substituting, combining, adapting, altering, expanding, eliminating, rearranging, or reversing and then speculating on how this single change would have a ripple effect on other aspects of the problem or the situation at hand.

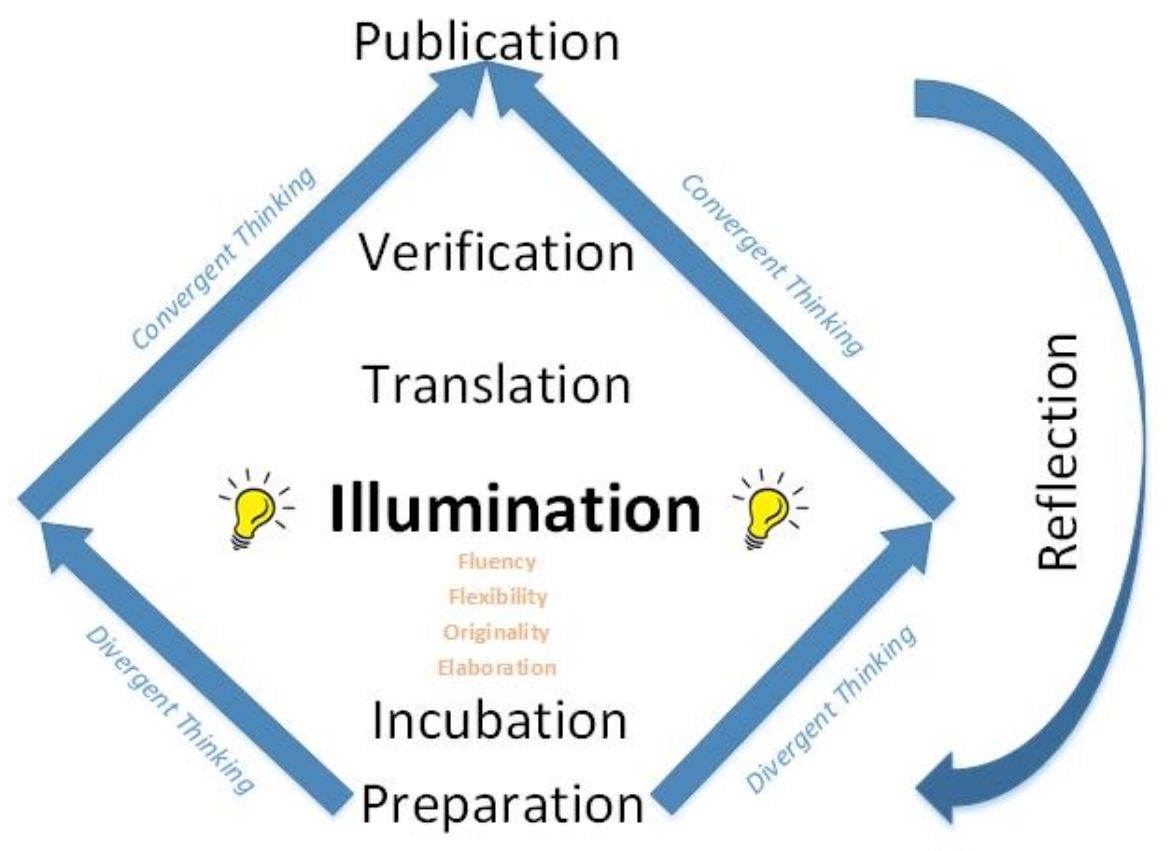

Figure 3. The creativity diamond

However, these stages are related only with the Divergent Thinking stage, where the individuals work in combination with conscious and unconscious processing generating what we call the fractal of the creative process. It means that the creative process not only happens in a cyclic movement, but also has recursive moments. Deciding to give a name to a variable is a little c creative moment, compared to a capital C creative moment like "defining Fuchsian groups" by Henri Poincaré for example.

At the climax of the divergent thinking process, the Illumination is an inflection point which allows the creative process to converge, leading to the actual production of something (an object, a story, a formal proof, a software...) outside the creator's brain, that can be shared with others and confronted with their points of views. Ideas can then be externalized and translated into things.

\section{Results and Examples}

Experiments were short (less than 4 hours) and not with an audience of the target group of primary and secondary students, rather their teachers and teacher trainers, but some conclusions can nevertheless be drawn, from the software produced and collected and from the oral exchanges which are only summarized here. First, although technology is lurking behind the scene and does promote advanced creative mathematical thinking, a basic unplugged version is doing the job of fostering creative mathematical thinking and might be preferable as an introduction: Paper printed versions were preferred even though the electronic versions allowed for electronic color picking or precise measurements. This is the sign of the conceptual nature of the pieces: something begs to be understood in these pictures and to be faithful to the actual artwork is not the challenge. In this paper, we present productions around Julio Le Parc artworks Ball, Composition, Sectors, the Railway illusion, the Coffee Bean illusion, which are examples of what was done on other pieces, climbing from confusion to subjective descriptions and finally to formalized algorithm. 
The tasks given to users of gradually describing the artwork led them to more and more precise terms and algorithms when the protocol was followed, but, as teacher-trainers often observe, many participating teachers took the instructions as a student's guide to be followed by the students and not by them, refusing the homology of the situation, avoiding the several steps scaffolding.

Based on the typical example of the modeling of a variation on Ball by Julio Le Parc (Figure. 4), we can describe the different levels of descriptions that went into different generic stages, following the diamond of creativity model (See Figure. 3) and the cycle of modeling: "I don't understand the question, can you please repeat, I don't see anything" (preparation); "I see a large square with a yellow cross in it" (elaboration on the preparation); "there are colors, disks and squares" (identifications of the relevant variables); "the disks are on the squares, they are both periodically colored and arranged in a table" (identification of preliminary relations between the variables); "the colors of the disks are always the same on a diagonal, likewise the colors of the squares along another diagonal" (elaboration on the identification of relations); some silent or vocal incubation; until the Ahah moment where the relationship between the variables color/shape/position springs to mind : "the color of a disk depends on the sum of its coordinates, the color of a square on their difference". Then the user enters a convergent phase, she can now try to translate, explain this crucial relationship and implement this algorithm using whether colored paper clips or a software of her choice. In some cases, amounting to 11 collective productions on diverse proposals, participants went as far as programming these algorithms (see Figure. 5-6).

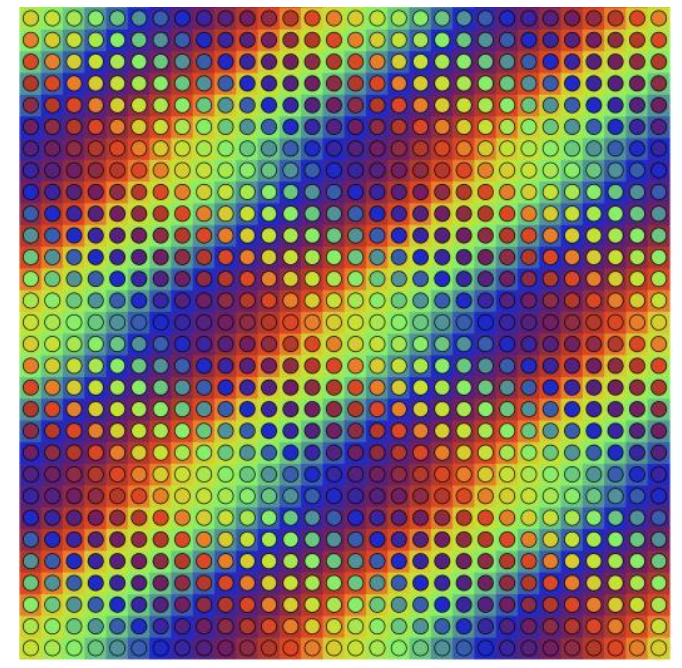

Figure 4. Variation on Ball by Julio Le Parc

In the Railway illusion (Figure 5 left), the illustrated implementation uses the spreadsheet containing geometric formulae, using translation and symmetry. It has been scaffolded on an example, not related to the Railway but using the geometric spreadsheet. This example has to be modified and generalized in order to reach the final goal of reproducing the visual effect. Meanwhile, the users got the crucial addition, with respect to a static reproduction, of an interactive exploration of the space of configurations (density, size and angle of lines and segments). Technically, each individual segment can be identified in the spreadsheet, which is not the case of the Coffee Bean illusion (Figure. 5 right) implementation: It was based on a rather sophisticated double loop of a translated and rotated base image, producing a depth two list of rotated and translated images. There are only four objects in this mesmerizing figure, two points, corners of an image, and this large list. This is a complex and abstract "one-liner" solution. A more concrete solution is to use the spreadsheet for explicitly unfolding a loop at each step, being able to understand the relationships between the objects. Using the spreadsheet is a good pedagogical middle-step in building the notion of loop and sequence (Haspekian-Bruillard 2008). It can be scaffolded on 1-loop and then 2-loop examples leading to more abstract constructs such as this Coffee Bean type of nested loops associated with a Cartesian product configuration space. 


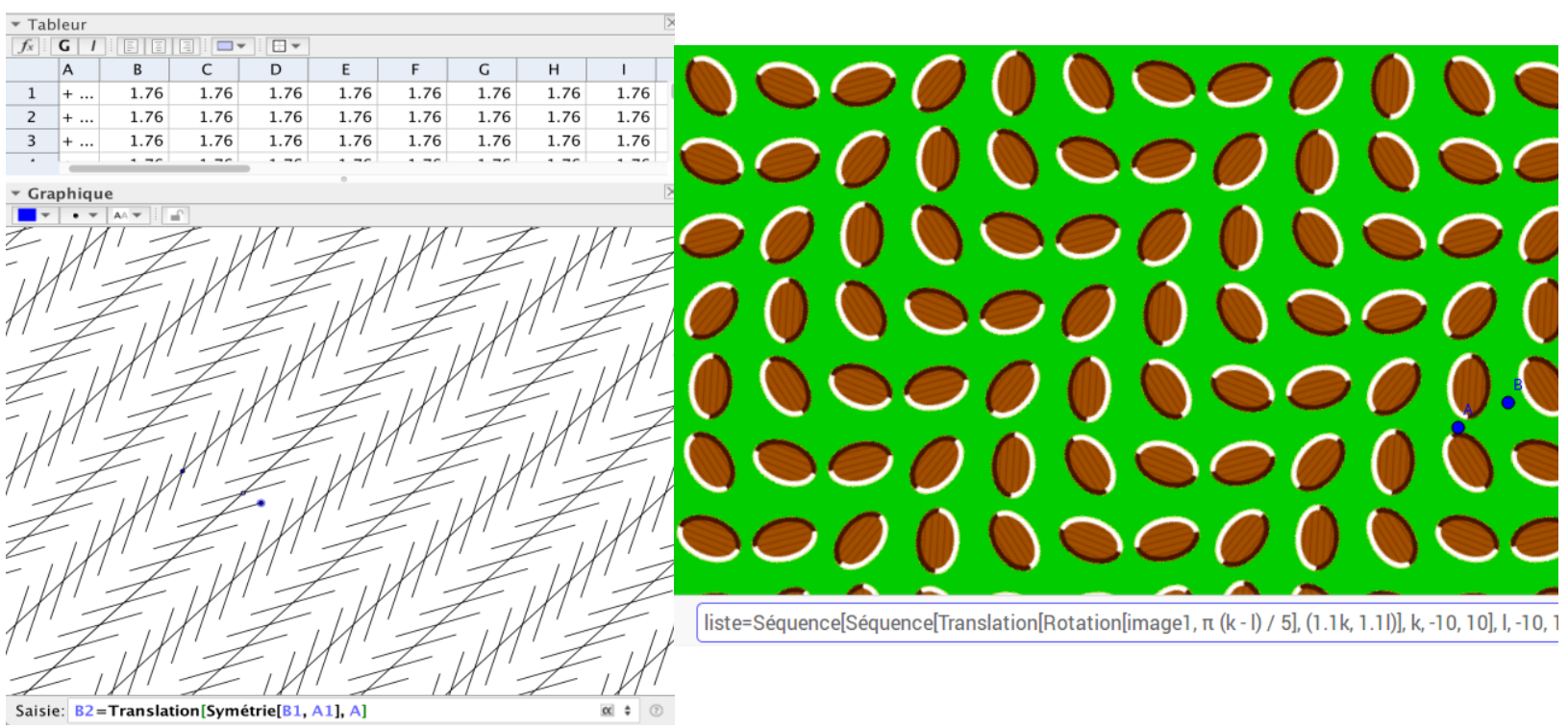

Figure 5. The Railway respectively Coffee Bean illusions based on a scaffolding of the use of the geometric spreadsheet (each cell contains a segment) resp. of sequences (loops) in Geogebra producing a large list.
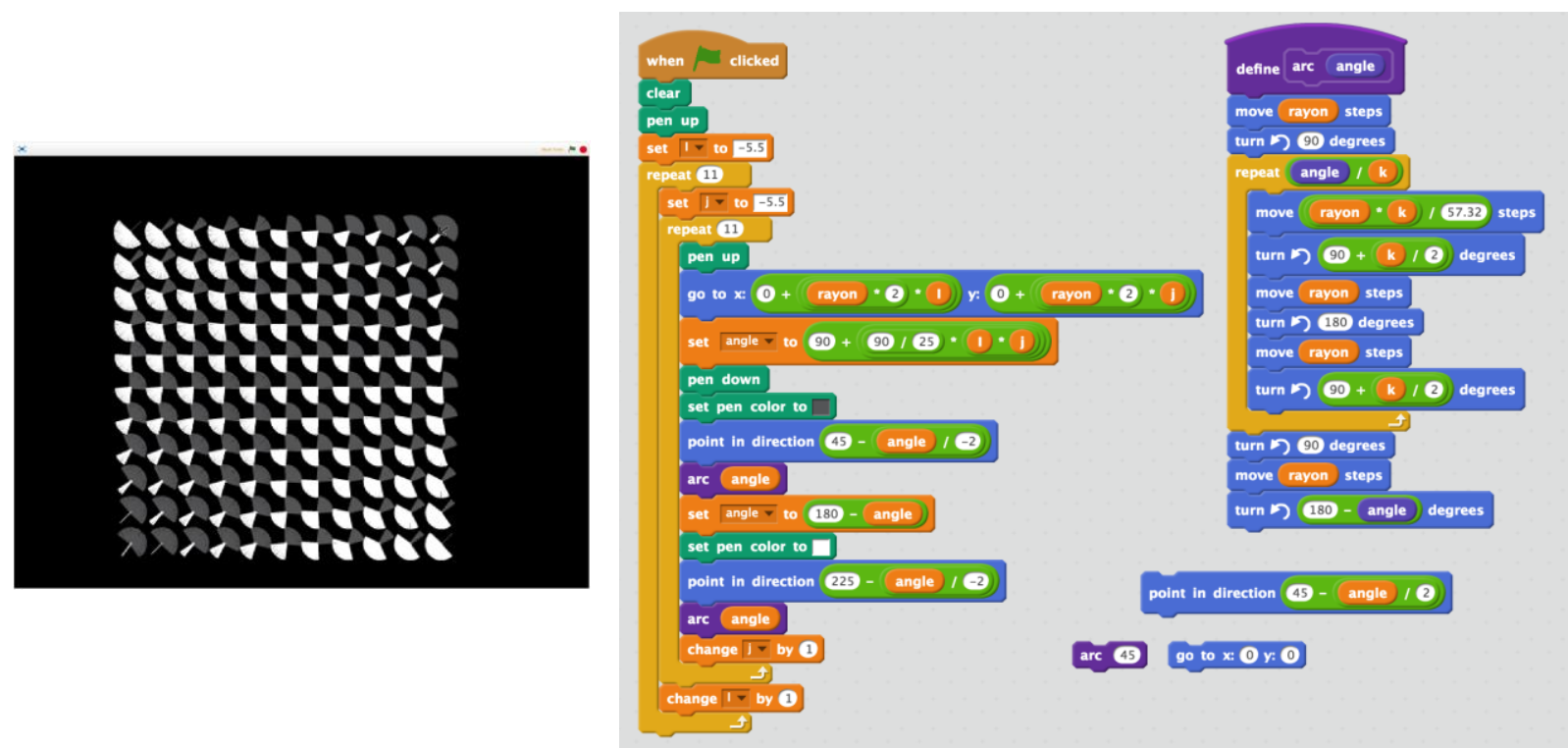

Figure 6. Given the angle construct, Julio Parc's Sector can be modeled with two nested loops.

In the Julio Le Parc Sector artwork (Figure. 6 for an implementation in Scratch), the user is presented the construct drawing an angular arc of a given angle. Then he is supposed to use it in two nested loops in order to draw grey and white sectors. Their angles increase linearly in each line y and each column $\mathrm{x}$, and at each point $(\mathrm{x}, \mathrm{y})$ add to a flat angle: therefore it can be modeled by $\pi / 2(1 \pm \mathrm{xy})$

\section{Conclusion}

The Op'Art movement is a goldmine and a good excuse for beautiful mathematical models without "the gorgeous trappings of painting or music", that is to say without the complexity of real life phenomena, as a first step in the direction of opening a scientific eye onto what surrounds us, to understand it, to model it, to be able to explain it on the phone, to simulate it on a computer and to 
finally see mathematics for what it is, a powerful tool that, with the due effort, unleashes creativity to grasp the world around us.

«Mathematics, rightly viewed, possesses not only truth, but supreme beauty, a beauty cold and austere, like that of sculpture, without appeal to any part of our weaker nature, without the gorgeous trappings of painting or music, yet sublimely pure, and capable of a stern perfection such as only the greatest art can show.»

Bertrand Russel

\section{References}

[1] Bulf, C., Mathé, A.-C. and Mithalal, J. (2015), Langage, résolution de problèmes et construction de connaissances en géométrie, Recherche en Didactique des Mathématiques, 35, p. 1.

[2] Chaachoua, H. (1997), Fonctions du dessin dans l'enseignement de la géométrie dans l'espace. Etude d'un cas : la vie des problèmes de construction et rapports des enseignants à ces problems, PhD Thesis, Université Joseph Fourier, Grenoble.

[3] Duval, R. (2005), Les conditions cognitives de l'apprentissage de la géométrie : développement de la visualisation, différenciation des raisonnements et coordination de leurs fonctionnements, Annales de Didactique et de Sciences Cognitives, 10, p. 5-53.

[4] El-Demerdash, M., Mercat, Ch., Trgalová, J., Essonnier, N. and Lealdino Filho, P., (2016), Design and evaluation of digital resources to enhance Creative Mathematical Thinking in a biomathematics context, Bulletin of the IEEE Technical Committee on Learning Technology. 18, 1, p. 6-9.

[5] Guilford, J. P. (1950), Creativity, American Psychologist, 5, 444-454.

[6] Haspekian, M. and Bruillard, E. (2008), What role does mathematics play in students' spreadsheet competencies? A case study of French students skills in a vocational school, In D. Benzie \& M. Iding (Eds.) Proceedings of Informatics, Mathematics and ICT, Boston, USA: College of Computer and Information Science Northeastern University.

[7] Houdement, C. and Kuzniak, A. (2006), Paradigmes géométriques et enseignement de la géométrie, Annales de Didactique et de Sciences Cognitives, 11, p. 175-193.

[8] Huguet, P. and Régner, I. (2007), Stereotype threat among schoolgirls in quasi-ordinary classroom circumstances, Journal of Educational Psychology, 99, 545-560

[9] Kitaoka, A. (2014), Visual illusion in ARTPOP and pop art, Japanese Journal of Psychonomic Science, 32, 2, p. 232-234.

[10] Laborde, C. and Capponi, B. (1994), Cabri-géomètre constituant d'un milieu pour l'apprentissage de la notion de figure géométrique, Recherches en Didactique des Mathématiques, 14, 1, p. 165210.

[11] Laborde, C. and Sträÿer, R. (2010), Place and use of new technology in the teaching of mathematics : ICMI activities in the past 25 years, ZDM - The International Journal on Mathematics Education, 42, 1, p.121-133.

[12] Mithalal, J. (2010), Instrumental deconstruction and dimensional deconstruction in 3D dynamic geometry, PhD Thesis, LIG, Université Joseph Fourier, Grenoble.

[13] Osterrieth, P. A. (1944), Le test de copie d'une figure complexe: Contribution à l'étude de la perception et de la mémoire, Archives de Psychologie, 30, p. 286-356.

[14] Prediger, S., Bikner-Ahsbahs, A., \& Arzarello, F. (2008), Networking strategies and methods for connection theoretical approaches: First steps towards a conceptual framework, ZDM - The International Journal of Mathematics Education, 40, 2, p. 165-178. 
[15] Rey, A. (1941), L'examen psychologique dans les cas d'encéphalopathie traumatique, Archives de Psychologie, 28, p. 286-340.

\section{Authors}

Christian Mercat, S2HEP EA4148, IREM, ESPE, University of Claude Bernard - Lyon 1, Lyon (France), christian.mercat@univ-lyon1.fr

Pedro Lealdino Filho, S2HEP EA4148, IREM, ESPE, University of Claude Bernard - Lyon 1, Lyon (France), pedrolealdino@gmail.com

Mohamed El-Demerdash, S2HEP EA4148, IREM, ESPE, University of Claude Bernard - Lyon 1, Lyon (France), and Menoufia University, Shibīn Al Kawm (Egypt), m_eldemerdash70@yahoo.com

\section{Acknowledgement}

The research leading to these results has received funding from the EU 7th Framework Programme (FP7/2007-2013) under grant agreement $\mathrm{n}^{\circ} 610467$ - project "M C Squared", http://mc2-project.eu. The C-book technology is based on the widely used Freudenthal Institute's DME portal and is being developed by a consortium of nine partner organizations, led by CTI\&Press 'Diophantus'. This publication reflects only the authors' views and the European Union is not liable for any use that may be made of the information contained therein. 